DESY 06-073

ISSN 0418-9833

hep-ph/0607201

July 2006

\title{
Heavy-quark QCD vacuum polarisation function: analytical results at four loops
}

\author{
Bernd A. Kniehl, Anatoly V. Kotikov* \\ II Institut für Theoretische Physik, Universität Hamburg, \\ Luruper Chaussee 149, 22761 Hamburg, Germany
}

5 June 2006

\begin{abstract}
Fully analytical results for the first two moments of the heavy-quark vacuum polarisation function at four loops in quantum chromo-dynamics are presented.

PACS: 12.38.-t, 12.38.Bx, 13.66.Bc, 13.85.Lg
\end{abstract}

*Permanent address: Bogoliubov Laboratory of Theoretical Physics, Joint Institute for Nuclear Research, 141980, Dubna (Moscow region), Russia. 


\section{Introduction}

During the past several years, there has been significant progress in the determination of the values of the strong-coupling constant $\alpha_{s}$ at the $Z$-boson mass scale and the heavyquark masses $m_{h}$ in perturbative quantum chromo-dynamics (QCD). The matching relations which determine the finite discontinuities of $\alpha_{s}$ at the heavy-quark thresholds in the modified minimal-subtraction $(\overline{\mathrm{MS}})$ scheme and are necessary for a precise determination of $\alpha_{s}$ from a global data fit were recently obtained in semi-analytical [1] and analytical 2 form at the four-loop level. Furthermore, there is significant progress in multi-loop technology aiming towards an analytical evaluation of the QCD $\beta$ function at five loops.

In order to precisely determine heavy-quark masses with the help of QCD sum rules [3, it is necessary to know in detail the heavy-quark contribution to the QCD vacuum polarisation function. The most important ingredients for this analysis are the lowest moments of the polarisation function (see Ref. 4]). Indeed, it was found that the first moment is best suited for this analysis, since it has the weakest dependence on nonperturbative effects and the details of the threshold region, thus leading to the smallest theoretical uncertainty [5].

At the four-loop level, the study of the first two moments has been started in Ref. 6] with calculations of diagrams including two internal loops of massive and massless quarks coupled to gluons. The results in Ref. 6] contain two numerical constants, denoted as $N_{10}$ and $N_{20}$, which were not expressed in terms of basic transcendental numbers. In the meantime, the corresponding Feynman diagrams have been calculated analytically 78 . The terms proportional to $\alpha_{s}^{j} n_{l}^{j-1}$, where $n_{l}$ in the number of light quarks, are known in all orders $j$ of perturbative QCD [9]. References [1011] contain the complete four-loop contributions to the first two Taylor coefficients from non-singlet diagrams. The singlet contributions were studied in Refs. 1112 .

The results of Refs. 1011] contain some four-loop tadpoles which are only given in numerical form. The purpose of this Letter is to evaluate them in terms of standard transcendental numbers so as to represent the first two moments of the heavy-quark vacuum polarisation function at four loops in completely analytical form.

The content of this Letter is as follows. Section 2 contains the basic formulae. In Section 3, we present our analytic results for the tadpoles that, in Refs. [1013], are called $T_{54}, T_{62}$, and $T_{91}$ as well as for the first two moments of the heavy-quark vacuum polarisation at four loops in QCD. A brief summary is given in Section 4.

\section{Approach}

Since the vector current $j^{\mu}(x)=\bar{\Psi}(x) \gamma^{\mu} \Psi(x)$ constructed from the heavy-quark field $\Psi(x)$ is conserved, the correlator,

$$
\begin{aligned}
\Pi^{\mu \nu}(q) & =i \int d x e^{i q x}\left\langle 0\left|T j^{\mu}(x) j^{\nu}(0)\right| 0\right\rangle \\
& =\left(-q^{2} g^{\mu \nu}+q^{\mu} q^{\nu}\right) \Pi\left(q^{2}\right)
\end{aligned}
$$


can be expressed in terms of a single scalar function $\Pi\left(q^{2}\right)$. The latter is of great phenomenological interest because it is related to the experimental observable

$$
\begin{aligned}
R(s) & =\frac{\sigma\left(e^{+} e^{-} \rightarrow \text { hadrons }\right)}{\sigma\left(e^{+} e^{-} \rightarrow \mu^{+} \mu^{-}\right)} \\
& =12 \pi \operatorname{Im} \Pi(s+i \epsilon),
\end{aligned}
$$

where $s$ is the square of the $e^{+} e^{-}$centre-of-mass energy. Equation (2) is equivalent to an infinite number of equalities between experimental moments,

$$
M_{n}^{\exp }=\int \frac{d s}{s^{n+1}} R(s),
$$

and their theoretical counterparts

$$
M_{n}^{\text {th }}=\frac{9 e_{h}^{2}}{4}\left(\frac{1}{4 \bar{m}_{h}^{2}}\right)^{n} \bar{C}_{n},
$$

where $e_{h}$ is the electric-charge quantum number of the heavy quark, $\bar{m}_{h}=m_{h}\left(\bar{m}_{h}\right)$, and $\bar{C}_{n}$ are the coefficients of the Taylor expansion

$$
\Pi\left(q^{2}\right)=\frac{3 e_{h}^{2}}{16 \pi^{2}} \sum_{n \geq 0} \bar{C}_{n} \bar{z}_{n}
$$

in $\bar{z}=q^{2} /\left(4 \bar{m}_{h}^{2}\right)$. The perturbative calculation of

$$
\bar{C}_{n}=\bar{C}_{n}^{(0)}+\frac{\alpha_{s}}{\pi} \bar{C}_{n}^{(1)}+\left(\frac{\alpha_{s}}{\pi}\right)^{2} \bar{C}_{n}^{(2)}+\left(\frac{\alpha_{s}}{\pi}\right)^{3} \bar{C}_{n}^{(3)}+\cdots
$$

where $\alpha_{s}=\alpha_{s}\left(\bar{m}_{h}^{2}\right)$, only involves tadpole diagrams and is, therefore, considerably simpler than the one of $\Pi\left(q^{2}\right)$, which has a complicated dependence on $\bar{z}$. Here, it is understood that $\alpha_{s}(\mu)$ and $m_{h}(\mu)$ are defined in the $\overline{\mathrm{MS}}$ renormalisation scheme, which is based on dimensional regularisation with $D=4-2 \epsilon$ space-time dimensions and 't Hooft mass scale $\mu$.

Thanks to the strong hierarchy among the quark masses, one may split the number $n_{f}=n_{l}+n_{h}$ of quark flavours into $n_{h}=1$ massive ones and $n_{l}$ massless ones. In the following, we keep the variable $n_{h}$ generic.

\section{Results}

In our previous paper [8], we introduced a technique that allows one to analytically evaluate a large class of four-loop tadpole diagrams with one non-zero mass. Specifically, the considered diagrams are transformed into integral representations whose integrands contain only one-loop tadpoles with new propagators having masses that depend on the variables of integration. This technique is based on the differential equation method [14. A similar technique was applied to certain types of two-loop diagrams in Ref. [15]. 


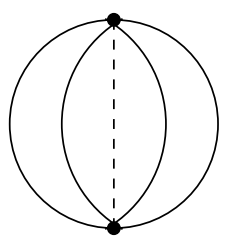

$T_{54}$

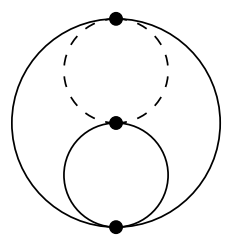

$T_{62}$

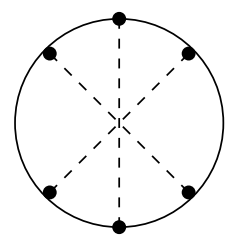

$T_{91}$

Figure 1: Massive four-loop tadpole diagrams $T_{54}, T_{62}$, and $T_{91}$.

To illustrate the usefulness of this technique, in Ref. [8], we also evaluated the tadpoles that are denoted in Ref. [6] as $N_{10}$ and $N_{20}$. The finite parts of our results agree with the numerical results of Ref. [6] and the analytical ones of Ref. [7], while our analytical results for the $O(\epsilon)$ terms are new.

These results together with those from Ref. 2] offer us the opportunity to obtain analytical results for terms of the expansion in $\epsilon$ of the master integrals denoted as $T_{54}$, $T_{62}$, and $T_{91}$ in recent papers [1013] for which only numerical values have been available so far. The corresponding Feynman diagrams are depicted in Fig. 1. Specifically, $T_{62}$ can be extracted from $N_{10}$ [8] using Eqs. (16) and (18) of Ref. [6]. This result can then be used in combination with $T_{91}$ [2] to extract $T_{54}$ from Eq. (50) of Ref. [13]. The results read

$$
\begin{aligned}
\frac{T_{54}}{N}= & -\frac{1}{\epsilon^{4}}-\frac{9}{2 \epsilon^{3}}-\frac{415}{36 \epsilon^{2}}-\frac{4991}{216 \epsilon}-\frac{89383}{1296}+\epsilon\left(-\frac{3367679}{7776}+\frac{1792}{9} \zeta(3)\right) \\
& +\epsilon^{2}\left(-\frac{137735095}{46656}-\frac{4352 \pi^{4}}{135}+\frac{68992}{27} \zeta(3)+\frac{1024}{3} b_{4}\right) \\
& +\epsilon^{3}\left(-\frac{4908181487}{279936}-\frac{167552 \pi^{4}}{405}+\frac{17408 \pi^{4}}{45} \ln 2+\frac{1602496}{81} \zeta(3)\right. \\
& \left.-\frac{87296}{3} \zeta(5)+\frac{39424}{9} b_{4}+4096 b_{5}\right)+O\left(\epsilon^{4}\right), \\
\frac{T_{62}}{N}= & \frac{3}{2 \epsilon^{4}}+\frac{4}{\epsilon^{3}}+\frac{38}{\epsilon^{2}}+\frac{1}{\epsilon}\left(\frac{44}{3}+\frac{16}{3} \zeta(3)\right)-118-\frac{4 \pi^{4}}{15}+88 \zeta(3) \\
& +\epsilon\left(-1156-\frac{374 \pi^{4}}{45}+\frac{2152}{3} \zeta(3)+96 \zeta(5)+64 b_{4}\right) \\
& +\epsilon^{2}\left(-\frac{20938}{3}-\frac{710 \pi^{4}}{9}+\frac{2416 \pi^{4}}{45} \ln 2-\frac{8 \pi^{6}}{21}+\frac{12952}{3} \zeta(3)\right. \\
& \left.+\frac{64}{3} \zeta^{2}(3)-2400 \zeta(5)+704 b_{4}+512 b_{5}\right)+O\left(\epsilon^{3}\right),
\end{aligned}
$$




$$
T_{91}=-\frac{53 \pi^{4}}{15} \ln 2+\frac{873}{2} \zeta(5)-48 b_{5}+O(\epsilon)
$$

where

$$
\begin{aligned}
& b_{4}=-\frac{1}{3}\left(\pi^{2}-\ln ^{2} 2\right) \ln ^{2} 2+8 \operatorname{Li}_{4}\left(\frac{1}{2}\right), \\
& b_{5}=\frac{1}{45}\left(5 \pi^{2}-3 \ln ^{2} 2\right) \ln ^{3} 2+8 \operatorname{Li}_{5}\left(\frac{1}{2}\right),
\end{aligned}
$$

and $N=\Gamma^{4}(1+\epsilon)\left(m^{2}\right)^{-4 \epsilon}$ is a normalisation factor. The choice $N=1$ corresponds to the normalisation adopted in Ref. 13. The terms through $O\left(\epsilon^{2}\right)$ in Eq. (77) and those through $O(\epsilon)$ in Eq. (8) agree with Eqs. (38) and (42) in Ref. 13], respectively, while the other terms represent new results.

Equations (71)-(92) extend the set of four-loop tadpole master integrals with one nonvanishing mass known in fully analytical form, and represent essential ingredients for high-precision predictions of numerous observables of current phenomenological interest. After changing the overall normalisation, we find agreement with the numerical values given in Ref. [7]. We note in passing that also the other master integrals presented in Ref. 7] can be written in a more compact form if the combinations $b_{4}$ and $b_{5}$ of Eq. (10) are introduced.

Equation (9) is the key result of Ref. 2] and a crucial ingredient for the four-loop matching condition of $\alpha_{s}(\mu)$ at the heavy-quark thresholds [12]. Equations (77)-(9]) are the missing links for a fully analytical representation of the moments $\bar{C}_{0}^{(3)}$ and $\bar{C}_{1}^{(3)}[10$, which is the main goal of this paper.

Our results for $\bar{C}_{0}^{(3)}$ and $\bar{C}_{1}^{(3)}$ read

$$
\begin{aligned}
\bar{C}_{0}^{(3)}= & n_{l}^{2}\left(\frac{17897}{69984}-\frac{31}{162} \zeta(3)\right)+n_{l} n_{h}\left(\frac{7043}{34992}+\frac{49 \pi^{4}}{12960}\right. \\
& \left.-\frac{127}{324} \zeta(3)-\frac{1}{36} b_{4}\right)+n_{h}^{2}\left(\frac{610843}{2449440}-\frac{661}{2835} \zeta(3)\right) \\
& +n_{l}\left(-\frac{71629}{46656}+\frac{8533 \pi^{4}}{116640}-\frac{21343}{3888} \zeta(3)-\frac{25}{324} b_{4}\right) \\
& +n_{h}\left(-\frac{83433703}{8164800}+\frac{14873 \pi^{4}}{18225}-\frac{14509529}{340200} \zeta(3)+\frac{5}{3} \zeta(5)\right. \\
& \left.-\frac{7091}{810} b_{4}\right)-\frac{5266559}{466560}-\frac{702959 \pi^{4}}{699840}-\frac{1289 \pi^{4}}{3645} \ln 2 \\
& +\frac{1688407}{12960} \zeta(3)-\frac{89}{24} \zeta(5)-\frac{313}{1944} b_{4}-\frac{328}{81} b_{5}, \\
\bar{C}_{1}^{(3)}= & n_{l}^{2}\left(\frac{42173}{98415}-\frac{112}{405} \zeta(3)\right)+n_{l} n_{h}\left(\frac{262877}{787320}+\frac{1421 \pi^{4}}{174960}\right. \\
& \left.-\frac{38909}{58320} \zeta(3)-\frac{29}{486} b_{4}\right)+n_{h}^{2}\left(\frac{163868}{295245}-\frac{3287}{7290} \zeta(3)\right)
\end{aligned}
$$




$$
\begin{aligned}
& +n_{l}\left(-\frac{9338899}{2099520}+\frac{372689 \pi^{4}}{839808}-\frac{48350497}{1399680} \zeta(3)-\frac{4793}{58320} b_{4}\right) \\
& +n_{h}\left(-\frac{27670774337}{1414551600}+\frac{1447057 \pi^{4}}{765450}-\frac{95617883401}{943034400} \zeta(3)\right. \\
& \left.+\frac{128}{27} \zeta(5)-\frac{348701}{17010} b_{4}\right)-\frac{5397779543}{146966400}-\frac{2653167371 \pi^{4}}{881798400} \\
& -\frac{359687 \pi^{4}}{229635} \ln 2+\frac{17554601717}{32659200} \zeta(3)-\frac{3655}{10206} \zeta(5)-\frac{84951877}{2449440} b_{4} \\
& -\frac{127480}{5103} b_{5} .
\end{aligned}
$$

These results agree with the semi-analytical ones presented in Refs. 1011.

As the authors of Refs. 1011, we conclude that the inclusion of the new four-loop corrections changes the actual values of the charm- and bottom-quark masses derived from QCD sum rules only very little, while it strongly decreases their theoretical uncertainties: approximately by factors of three and four in the case of charm and bottom, respectively.

\section{Conclusion}

We presented analytical results for the four-loop tadpole master integrals involving one non-vanishing mass that are called $T_{54}, T_{62}$, and $T_{91}$ in the recent literature [10 13], for which only numerical values had been available so far. They represent essential ingredients for high-precision predictions of a number of observables of current phenomenological interest. As such an application, we considered the vacuum polarisation induced by a heavy quark in the four-loop approximation of QCD and expressed the first two coefficients of its Taylor expansion in the ratio of virtuality to heavy-quark mass in fully analytical form. The introduction of the characteristic combinations $b_{4}$ and $b_{5}$ of basic transcendental numbers turned out the be useful in order to compactify the expressions. Other applications include the four-loop QCD correction to the electroweak $\rho$ parameter, which has just been presented in semi-analytic form [16].

\section{Acknowledgements}

We are grateful to O.I. Onishchenko for useful communications and technical advice related to the programming language Mathematica, and to O.L. Veretin for checking some of our formulae and for technical advice related to the PSLQ program [17, which helped us in checking the $O(\epsilon)$ terms of the four-loop tadpoles $N_{10}$ and $N_{20}$. A.V.K. was supported in part by the RFBR Foundation through Grant No. 05-02-17645-a and the HeisenbergLandau-Programm. This work was supported in part by BMBF Grant No. 05 HT4GUA/4 and HGF Grant No. NG-VH-008. 


\section{References}

[1] Y. Schröder, M. Steinhauser, JHEP 0601 (2006) 051;

K.G. Chetyrkin, J.H. Kühn, C. Sturm, Nucl. Phys. B 744 (2006) 121.

[2] B.A. Kniehl, A.V. Kotikov, A.I. Onishchenko, O.L. Veretin, Report No. DESY 06074 .

[3] V.A. Novikov, L.B. Okun, M.A. Shifman, A.I. Vainshtein, M.B. Voloshin, V.I. Zakharov, Phys. Rept. 41 (1978) 1;

L.J. Reinders, H. Rubinstein and S. Yazaki, Phys. Rept. 127 (1985) 1.

[4] M.A. Shifman, A.I. Vainshtein, V.I. Zakharov, Nucl. Phys. B 147 (1979) 385;

M.A. Shifman, A.I. Vainshtein, V.I. Zakharov, Nucl. Phys. B 147 (1979) 448.

[5] J.H. Kühn, M. Steinhauser, Nucl. Phys. B 619 (2001) 588;

J.H. Kühn, M. Steinhauser, Nucl. Phys. B 640 (2002) 415 (Erratum).

[6] K.G. Chetyrkin, J.H. Kühn, P. Mastrolia, C. Sturm, Eur. Phys. J. C 40 (2005) 361.

[7] Y. Schröder, A. Vuorinen, JHEP 0506 (2005) 051.

[8] B.A. Kniehl, A.V. Kotikov, Phys. Lett. B 638 (2006) 531.

[9] A.G. Grozin, C. Sturm, Eur. Phys. J. C 40 (2005) 157.

[10] K.G. Chetyrkin, J.H. Kühn, C. Sturm, Report No. SFB/CPP-06-21, TTP06-15, hep-ph/0604234.

[11] R. Boughezal, M. Czakon, T. Schutzmeier, hep-ph/0605023.

[12] S. Groote, A.A. Pivovarov, Eur. Phys. J. C 21 (2001) 133;

J. Portolés, P.D. Ruiz-Femenía, J. Phys. G 29 (2003) 349;

J. Portolés, P.D. Ruiz-Femenía, Eur. Phys. J. C 24 (2002) 439.

[13] K.G. Chetyrkin, M. Faisst, C. Sturm, M. Tentyukov, Nucl. Phys. B 742 (2006) 208.

[14] A.V. Kotikov, Phys. Lett. B 254 (1991) 158;

A.V. Kotikov, Phys. Lett. B 259 (1991) 314;

A.V. Kotikov, Phys. Lett. B 267 (1991) 123;

E. Remiddi, Nuovo Cim. A 110 (1997) 1435.

[15] J. Fleischer, A.V. Kotikov, O.L. Veretin, Phys. Lett. B 417 (1998) 163;

J. Fleischer, A.V. Kotikov, O.L. Veretin, Nucl. Phys. B 547 (1999) 343;

B.A. Kniehl, A.V. Kotikov, A.I. Onishchenko, O.L. Veretin, Nucl. Phys. B 738 (2006) 306. 
[16] K.G. Chetyrkin, M. Faisst, J.H. Kühn, P. Maierhöfer, C. Sturm, Report No. SFB/CPP-06-24, TTP06-17, hep-ph/0605201;

Boughezal, M. Czakon, Report No. hep-ph/0606232

[17] H.R.P. Ferguson, D.H. Bailey, RNR Technical Report No. RNR-91-032;

H.R.P. Ferguson, D.H. Bailey, S. Arno, NASA Technical Report No. NAS-96-005. 